\title{
Charge Distributions of Crystal Field States
}

\author{
U. Walter* \\ II. Physikalisches Institut, Universität zu Köln, Federal Republic of Germany \\ Z. Phys. B - Condensed Matter 62, 299-309 (1986)
}

In Table 2, the wrong sign was given for the Stevens' factor $\theta_{2}$ for $\mathrm{Dy}^{3+}$. The correct value should read $\theta_{2}=-0.6349 \times 10^{-2}$.

\author{
U. Walter \\ II. Physikalisches Institut \\ Universität zu Köln \\ Zülpicher Strasse 77 \\ D-5000 Köln 41 \\ Federal Republic of Germany
}

* Present address: Materials Science and Technology Division, Argonne National Laboratory, Argonne, Illinois 60439 , USA 